Arch. Tierz., Dummerstorf 42 (1999) 6, 601-610

Aus dem Institut fur Tierzucht und Haustiergenetik der Universităt Göttingen

HORST BRANDT und BURCHHARD MÖLLERS

\title{
Inzuchtdepression bei Merkmalen der Fruchtbarkeit und der Gewichtsentwicklung beim Göttinger Miniaturschwein
}

\author{
Herrn Professor Dr. Peter Glodek zum 65. Geburtstag gewidmet
}

\begin{abstract}
Summary
Title of the paper: Inbreeding depression for litter traits and the development of growth in the Göttinger Minipig

A data set of 1191 litters from 282 sows and 6777 piglets weights from the Göttinger Minipig was analysed to estimate inbreeding depression for litter traits and the early growth rates up to an age of 12 month. The population of the Göttinger Minipig shows an average inbreeding of sows and piglets of about $10 \%$ with a standard deviation of $1.7 \%$ with a nearly normal distribution of the inbreeding coefficients in contrast to most other studies about inbreeding depression. There is no inbreeding depression observed for number of piglets born alive or born dead within a litter, neither for inbreeding of sows nor for inbreeding of litters. For average and individual birth weights the inbreeding of sows show a significant influence while the inbreeding of the litter is not significant. With a 10 percent increase of the inbreeding of sows a reduction on individual birth weight of 70 gram is observed $(70 \%$ of the phenotypic standard deviation). For the weight of piglets in the first 6 month both the inbreeding of sows and the inbreeding of litters show a significant effect. A 10 percent increase of inbreeding of sows or litters both leads to a reduction on weight within the first 6 month of 250 gram ( $20 \%$ of the phenotypic standard deviation.
\end{abstract}

Key Words: inbreeding depression, minipig, litter traits, development of weights

\section{Zusammenfassung}

Die Inzuchtdepression bei Wurfleistungsmerkmalen und der Gewichtsentwicklung von Ferkeln bis zum Alter von 12 Monaten wurde an der Population des Göttinger Minischweins an 1191 Würfen von 282 Sauen und Gewichten von 6777 Ferkeln untersucht. Im Gegensatz zu vielen anderen Studien über Inzuchtdepression sind die Inzuchtkoeffizienten des vorliegenden Datenmaterials normalverteilt mit einem Mittel von $10 \%$ und einer Standardabweichung von 1,7 \%. Für die Zahl der lebend bzw. tot geborenen Ferkel pro Wurf ist keine Inzuchtdepression zu beobachten. Weder die Inzucht der Sau noch die Inzucht des Wurfes zeigen auf diese Merkmale einen signifikanten Effekt. Auf das durchschnittliche und das individuelle Geburtsgewicht zeigt die Inzucht der Sau einen signifikanten Einfluß während die Inzucht des Wurfes nicht von Bedeutung ist. Mit einer Steigerung der Inzucht der Sau um $10 \%$ reduziert sich das Geburtsgewicht um 70 Gramm (70\% der phänotypischen Standarabweichung). Auf die Gewichtsentwicklung in den ersten 6 Lebensmonaten haben sowohl die Inzucht der Sau als auch die Inzucht des Wurfes einen signifikanten Effekt. Pro $10 \%$ Inzuchtsteigerung der Sau bzw. des Wurfes reduziert sich das Gewicht in den ersten 6 Lebensmonaten um ca. 250 Gramm (20\% der phänotypischen Standardabweichung).

Schlüsselwörter: Inzuchtdepression, Minischwein, Wurfleistungsmerkmale, Gewichtsentwicklung

Der heutige Markt für landwirtschaftliche Erzeugnisse tendiert zu einer immer höheren Anforderung an die Uniformität der Produkte. Beim Schwein spiegelt sich diese Forderung in den enger werdenden Grenzen im Schlachtgewicht ohne Preisabzüge in der Be- 
zahlung wider. Eine mögliche Zuchtstrategie zu mehr Uniformität könnten Inzuchtlinien sein, wenn nicht das für quantitative Merkmale bekannte Phänomen der Inzuchtdepression (FALCONER, 1989) zu einem Verlust in der allgemeinen Vitalität und Fertilität führen würde. In den Zuchtprogrammen für landwirtschaftliche Nutztiere ist es daher oft ein Ziel, Inzucht zu vermeiden. Auch bei der Erhaltung seltener, vom Aussterben bedrohter Rassen wird in den Erhaltungsprogrammen gezielt auf eine Minimierung der Inzucht geachtet.

In den heutigen modernen Schweinezuchtprogrammen werden die Basislinien für Dreioder Vierrassenkreuzungen oft in geschlossenen, kleinen Populationen mit Größen von nur 200 bis 1000 Sauen gehalten. Für solche kleinen Populationen ist langfristig mit einer Inzuchtsteigerung zu rechnen, und daher ist es von großer Bedeutung, das Ausmaß der Inzuchtdepression zu kennen. In dieser Untersuchung werden die Auswirkungen der Inzucht am Beispiel des Göttinger Minischweins, welches schon seit Ende 1960 als geschlossene Population gehalten wird (GLODEK und OLDIGS, 1981), auf Fruchtbarkeitsmerkmale und auf die Gewichtsentwicklung bis zum Alter von 12 Monaten dargestellt. Untersuchungen über Inzuchtdepressionen beim Schwein sind bisher nur aus gezielten Selektionsexperimenten (BERESKIN et al., 1968, 1970) oder bei kleinen lokalen Populationen, wie z. B. bei Iberischen Schweinelinien (TORO et al., 1988; SILIO et al., 1994) bekannt.

1.

Material und Methoden

Seit 1992 besteht neben der Basiszucht des Göttinger Minischweins im Versuchsgut Relliehausen der Universität Göttingen ein Vermehrungsbetrieb in Dänemark (Ellegaard Göttingen Minipig). Dieser Vermehrungsbetrieb wurde mittels Hysterektomie mit Tieren aus Relliehausen aufgebaut und wird als geschlossener Zuchtbetrieb geführt. Neben der Erfassung der üblichen Wurfleistungsmerkmale werden individuelle Ferkelgewichte bei der Geburt und dann in regelmäßigen Abständen von ca. 4 Wochen routinemäßig erhoben. In der vorliegenden Analyse wurden ausschließlich Daten aus dem Betrieb in Dänemark verwendet. Es konnten insgesamt 1191 Würfe von 282 Sauen und Gewichte von 6777 Ferkeln ausgewertet werden. Eine Verteilung der Wurfnummern über Jahre ist in Tabelle 1 dargestellt. In der Tabelle ist zu erkennen, daß erst ab 1994 dritte und höhere Wurfnummern vorhanden sind. Für die weitere statistische Analyse wurden die in Tabelle 1 dargestellten Wurfnummernklassen im Modell berïcksichtigt.

Tabelle 1

Verteilung der Wurfnummern über Jahre (Distribution of litters over years)

\begin{tabular}{|c|c|c|c|c|c|}
\hline \multirow[t]{2}{*}{ Jahre } & \multicolumn{4}{|c|}{ Wurfnummer } & \multirow[b]{2}{*}{ Summe } \\
\hline & 1 & 2 & 3 und 4 & $>4$ & \\
\hline 1993 & 40 & 6 & 0 & 0 & 46 \\
\hline 1994 & 41 & 51 & 52 & 1 & 145 \\
\hline 1995 & 51 & 65 & 103 & 64 & 283 \\
\hline 1996 & 55 & 33 & 86 & 102 & 276 \\
\hline 1997 & 53 & 61 & 54 & 93 & 261 \\
\hline 1998 & 42 & 25 & 51 & 62 & 322 \\
\hline Summe & 282 & 241 & 346 & 322 & 1191 \\
\hline
\end{tabular}


Als Merkmale der Gewichtsentwicklung wurde das Geburtsgewicht der Ferkel, die Gewichtsentwicklung in den ersten 6 Lebensmonaten (ohne Geburtsgewicht) und in den Lebensmonaten 6 bis 12 untersucht. Aufgrund der speziellen Fütterungsstrategie, nach der die Tiere individuell nach Bedarf gefüttert werden, ist es nicht sinnvoll die individuellen Gewichte direkt auszuwerten. Es wurde deshalb das Gewicht aller Wiegungen in den ersten 6 Lebensmonaten und in den Lebensmonaten 6 bis 12 als mittlere Abweichung von der durchschnittlichen Wachstumskurve aller Tiere in der Auswertung berücksichtigt. Nähere Einzelheiten zur Wachstumskurve und zu genetischen Parametern der Wachstumsentwicklung sind bei BRANDT et al. (1998) beschrieben. In Tabelle 2 ist die Verteilung der Merkmale der Gewichtsentwicklung über Jahre dargestellt. Aufgrund des Verkaufes von meist jungen Tieren aus dem Vermehrungsbetrieb in Dänemark ist nur noch von 1710 Tieren die Gewichtsentwicklung im Alter von 6 bis 12 Monaten vorhanden.

Tabelle 2

Verteilung der Einzelferkelgewichte über Jahre (Distribution of individual piglet weights over years)

\begin{tabular}{l|ccc}
\hline Jahre & Geburtsgewicht & Gewicht bis 6 Monate & Gewicht 6 bis 12 Monate \\
\hline 1993 & 176 & 171 & 78 \\
1994 & 913 & 857 & 389 \\
1995 & 1789 & 1650 & 497 \\
1996 & 1724 & 1605 & 447 \\
1997 & 1159 & 1023 & 299 \\
1998 & 1016 & 877 & 0 \\
\hline Summe & 6777 & 6183 & 1710 \\
\hline
\end{tabular}

Die Rohmittelwerte aller untersuchten Merkmale mit Standardabweichungen sind in Tabelle 3 zusammengestellt. Da die Gewichte bis 6 Monate und im Alter von 6 bis 12 Monaten als durchschnittliche Abweichung von der Wachstumskurve aller Tiere dargestellt sind, liegen die Mittelwerte für diese Merkmale erwartungsgemäß nahe bei Null. Die Inzuchtkoeffizienten der Sauen und Ferkel wurden unter Berücksichtigung aller verfügbaren Abstammungsinformationen (Daten ab 1970) berechnet. Der mittlere Inzuchtkoeffizient der Ferkel und der Sauen liegt bei ca. $10 \%$ mit einer Standardabweichung von 1,7 \%. Es bleibt anzumerken, daß die Inzuchtkoeffizienten für Sauen und für Ferkel in dem vorliegenden Datenmaterial nahezu normalverteilt sind, im Gegensatz zu vielen anderen Auswertungen, bei denen sehr viele nicht ingezüchtete Tiere mit wenigen Tieren mit In-

Tabelle 3

Anzahl an Beobachtungen, Rohmittelwerte und Standardabweichungen der untersuchten Merkmale (Number of observations, means and standard deviations for the analysed traits)

\begin{tabular}{lc|ccc}
\hline Merkmal & (Einheit) & Anzahl & Mittelwert & Standardabweichung \\
\hline Inzucht der Ferkel & $(\%)$ & 6777 & 9,96 & 1,78 \\
Inzucht der Sauen & $(\%)$ & 282 & 10,23 & 1,59 \\
Geburtsgewicht & $(\mathrm{g})$ & 6777 & 434 & 103 \\
Gewichte bis 6 Monate & $(\mathrm{g})$ & 6183 & 36 & 940 \\
Gewichte 6 bis 12 Monate & $(\mathrm{kg})$ & 1710 & 0,20 & 4,02 \\
Lebend geborene Ferkel & (pro Wurf) & 1191 & 6,4 & 2,07 \\
Lebend geborene männlich & (pro Wurf) & 1191 & 3,3 & 1,62 \\
Lebend geborene weiblich & (pro Wurf) & 1191 & 3,1 & 1,63 \\
Tot geborene Ferkel & (pro Wurf) & 1191 & 0,22 & 0,63 \\
Mittleres Geburtsgewicht & (g) & 1184 & 439 & 84 \\
\hline
\end{tabular}


zuchtkoeffizienten größer als Null verglichen werden (BERESKIN et al., 1968, 1970; TAKAHASHI et al., 1991).

In Abbildung 1 und Tabelle 4 sind die Verteilungen der Inzuchtkoeffizienten der Sauen und der Würfe mit Mittelwerten und Streuungen über Jahre dargestellt. Aus der Abbildung und der Tabelle wird deutlich, daß für das vorliegende Datenmaterial eine gute Trennung von Umwelt und Inzuchtdepression in der Auswertung möglich ist. Das von LYNCH und WALSH (1997) dargestellte Problem eines „Confoundings“ zwischen Generation bzw. Jahr und Inzuchtgrad ist hier nicht gegeben. Tabelle 4 zeigt, daß der mittlere Inzuchtkoeffizient der Sauen über Jahre tendenziell gesunken ist. Die Sauen aus dem Geburtsjahr 1995 zeigen eine größere Steubreite als alle anderen Sauen.

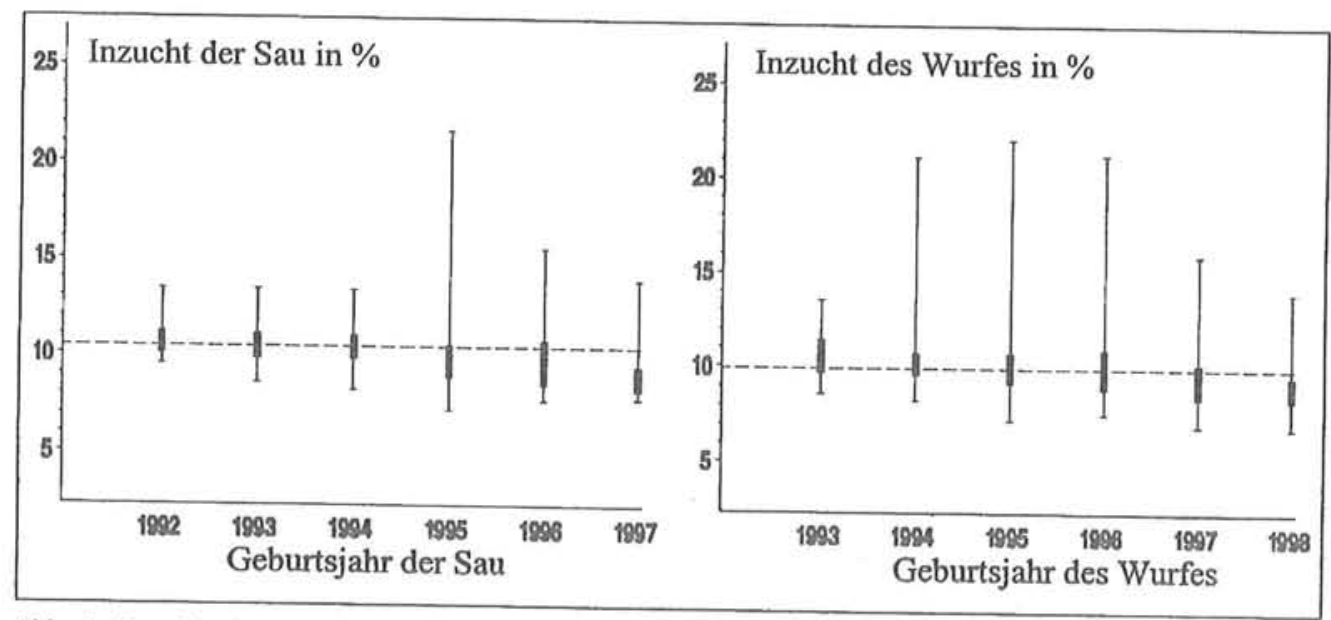

Abb. 1.: Boxplot der Inzucht der Sauen und der Würfe für Jahre (Boxplot of inbreeding of sows and litters for years)

Tabelle 4

Anzahl Sauen und durchschnittlicher Inzuchtkoeffizient mit Standardabweichung, Minimum und Maximum nach Geburtsjahr (Number of sows with average inbreeding coefficients, standard deviation, minimum and maximum by year of birth)

\begin{tabular}{l|ccccc}
\hline Geburtsjahr & Anzahl & Mittelwert & Standardabw. & Minimum & Maximum \\
\hline 1992 & 48 & 10,8 & 0,88 & 9,5 & 13,4 \\
1993 & 40 & 10,5 & 1,09 & 8,6 & 13,4 \\
1994 & 51 & 10,5 & 1,14 & 8,2 & 13,4 \\
1995 & 59 & 10,1 & 2,20 & 7,2 & 21,7 \\
1996 & 62 & 9,9 & 1,74 & 7,7 & 15,6 \\
1997 & 23 & 9,4 & 1,55 & 7,8 & 13,9 \\
\hline
\end{tabular}

In Abbildung 2 ist die Verteilung der Inzucht der Sauen und der Inzucht der Würfe dargestellt. Die Abbildung zeigt, daß es keine erkennbare Beziehung zwischen dem Inzuchtgrad der Sauen und dem der Würfe gibt. Es ist anzumerken, daß im Zuchtprogramm furr die Minischweine ein Paarungsplan zur Vermeidung von Inzucht aufgestellt wird. 


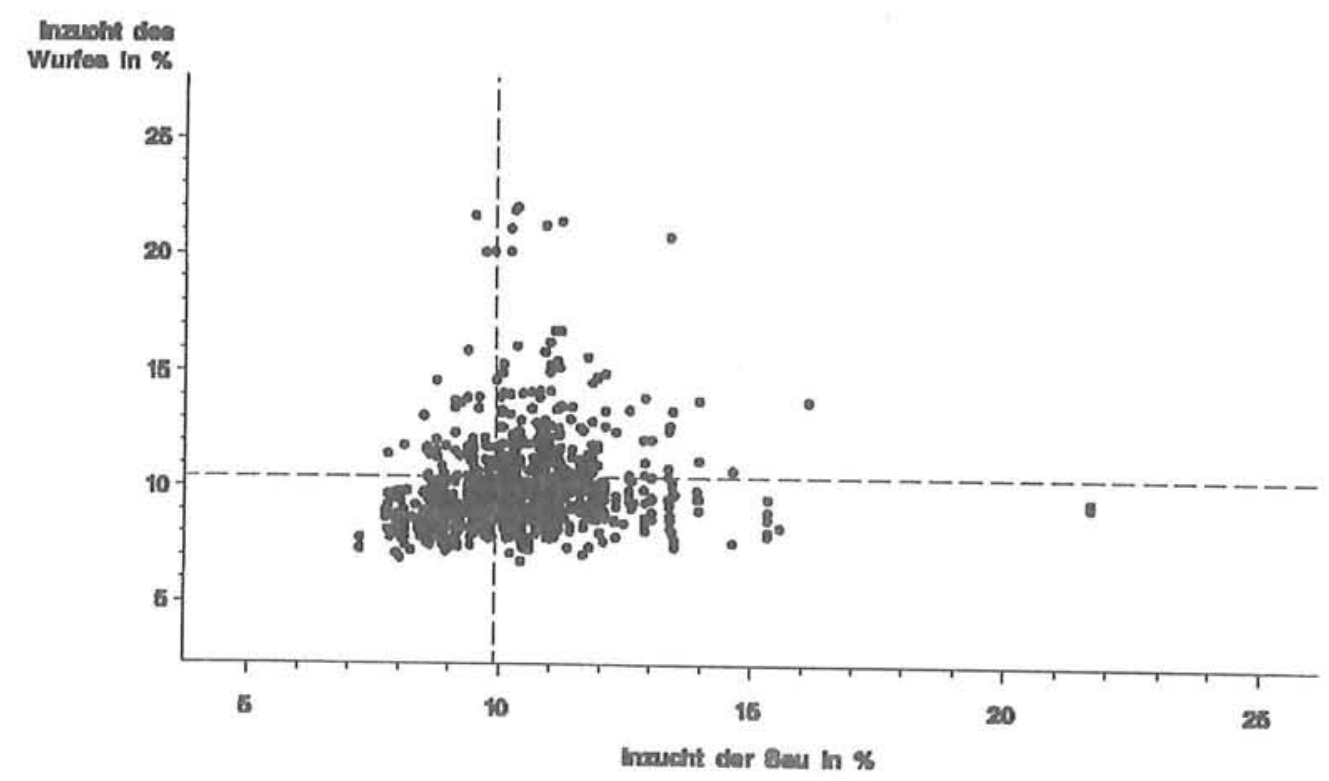
Abb. 2.: Verteilung der Inzucht der Würfe über Inzucht der Sauen (Distribution of inbreeding of sows and
inbreeding of litters)

Die Auswertung der Daten erfolgte mit Prozeduren des SAS Programmpaketes. Für die Wurfleistungsmerkmale wurden die Wurfnummer (Klassen siehe Tabelle 1) und das Jahr als fixe Effekte sowie die Inzucht des Wurfes und der Sau als Kovariable berücksichtigt. Zur Erkennung einer nichtlinearen Beziehung zwischen der Inzucht der Sauen und den Wurfleistungsmerkmalen wurde die Inzucht der Sau in einem weiteren Modell als fixer Einflußfaktor mit 8 Klassen berücksichtigt, wobei die Sauen nach ihrem Inzuchtkoeffizienten sortiert in Klassengrößen von ca. 35 bis 40 Sauen eingeteilt wurden. Die mittleren Inzuchtkoeffizienten der Sauen in den jeweiligen Klassen sind im Ergebnisteil in Abbildung 3 auf der X-Achse dargestellt.

Bei den Merkmalen der Gewichtsentwicklung wurde neben der Inzucht der Mütter und der Inzucht der Ferkel auch die Zahl der gesamt geborenen Ferkel im Wurf als Kovariable im Modell berücksichtigt. Als fixe Effekte wurden das Jahr und das Geschlecht ins Modell mit aufgenommen.

\section{Ergebnisse}

In Tabelle 5 sind die Signifikanzen der Effekte auf die Wurfleistungsmerkmale zusammengestellt. Bei der Zahl der lebend geborenen Ferkel und des durchschnittlichen Geburtsgewichtes sind signifikante Jahreseffekte zu beobachten. Die Wurfnummer wirkt sich erwartungsgemäß auf alle Merkmale mit Ausnahme der Zahl der tot geborenen Ferkel signifikant aus. Ein signifikanter Einfluß der Inzucht der Sauen oder des Wurfes läßt sich nur für das durchschnittliche Geburtsgewicht und bei der Berücksichtigung der Inzucht der Sau in Klassen als fixer Effekt beobachten. 
Tabelle 5

Signifikanz der untersuchten Effekte auf die Wurfleistungsmerkmale (Significance of effects on litter traits)

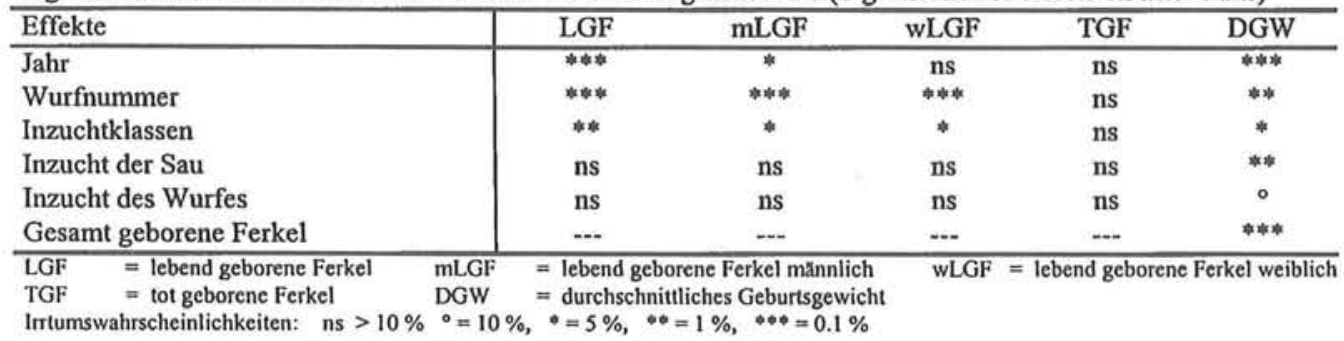

In Tabelle 6 sind die Signifikanzen der untersuchten Effekte auf die Merkmale der Gewichtsentwicklung zusammengefaßt dargestellt. Es sind auch bei diesen Merkmalen signifikante Jahreseffekte zu beobachten. Das Geschlecht übt ebenfalls einen signifikanten Einfluß auf die Merkmale aus, mit Ausnahme der Gewichtsentwicklung in den ersten 6 Lebensmonaten. Die Inzucht der Ferkel und der Sau sowie die Zahl der Ferkel im Wurf zeigen nur bei den Merkmalen bis zum Alter von 6 Monaten signifikante Einflüsse. Für die spätere Gewichtsentwicklung standen allerdings auch nur noch ein Viertel aller Tiere in der Analyse zur Verfügung.

Tabelle 6

Signifikanzen der untersuchten Effekte auf die Gewichtsentwicklung der Ferkel (Significance of effects on weights of piglets)

\begin{tabular}{|c|c|c|c|}
\hline & GGW & GEW06 & GEW12 \\
\hline Jahr & $* \psi$ & \#** & 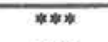 \\
\hline Geschlecht & $* * *$ & ns & $* * *$ \\
\hline Inzucht der Ferkel & $\circ$ & $\% * *$ & $\circ$ \\
\hline Inzucht der Sau & 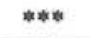 & $4 *$ & ns \\
\hline
\end{tabular}

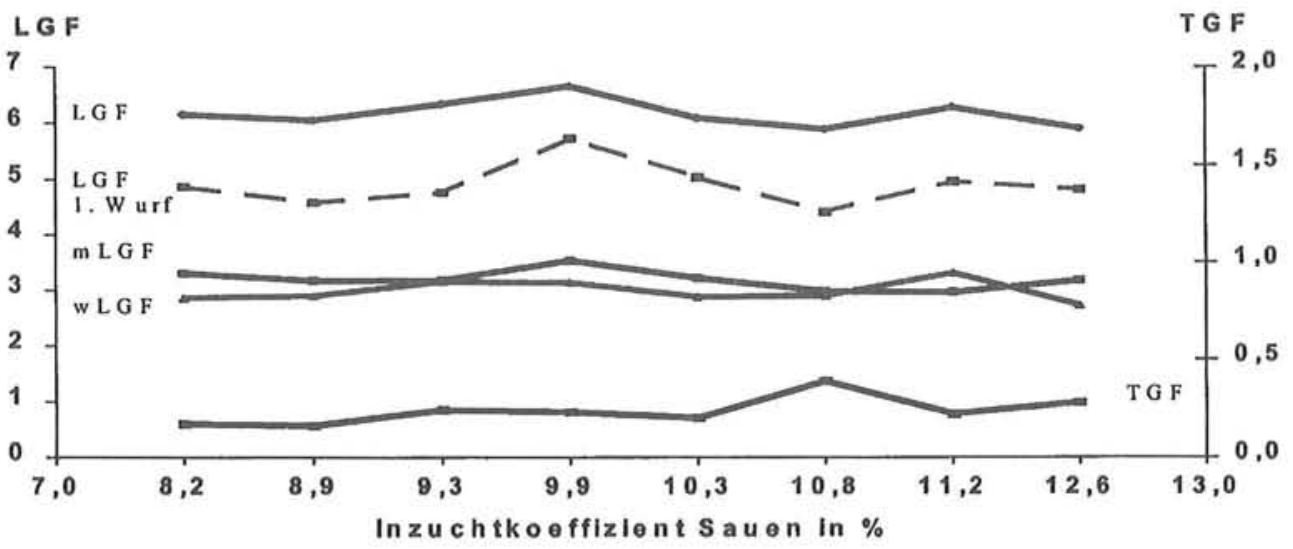

Abb. 3.: LSQ-Mittelwerte für Wurfleistungsmerkmale für Inzuchtklasssen der Sauen (Werte auf der X-Achse stellen die mittlere Inzucht der jeweiligen Klasse dar) (Least square means for litter traits for classes of inbreeding of sows) (values on the $\mathrm{x}$-axis represent the average inbreeding of sows within each class) 
In Abbildung 3 sind die LSQ-Mittelwerte für die Wurfleistungsmerkmale für die Inzuchtklassen der Sauen grafisch dargestellt. Obwohl der Effekt der Inzuchtklassen einen leicht signifikanten Einfluß auf die Zahl der lebend geborenen Ferkel (gesamt, männlich und weiblich) ausübt, ist kein Trend für eine Inzuchtdepression zu erkennen.

Die in der Abbildung 3 dargestellten Mittelwerte der Inzuchtklassen lassen weder eine lineare noch eine nichtlineare Abhängigkeit der Leistung vom Inzuchtkoeffizienten der Sauen erkennen.

In Abbildung 4 ist die Beziehung zwischen dem durchschnittlichen Geburtsgewicht der Ferkel und der Inzucht der Sauen in Form der LSQ-Mittelwerte für die Inzuchtklassen und auch der linearen Regression des Geburtsgewichtes auf die Inzucht der Sauen (gestrichelte Linie) grafisch dargestellt. In der Abbildung wird die signifikante lineare Beziehung zwischen der Inzucht der Sauen und dem Geburtsgewicht sowohl für alle Würfe als auch nur für erste Würfe deutlich.

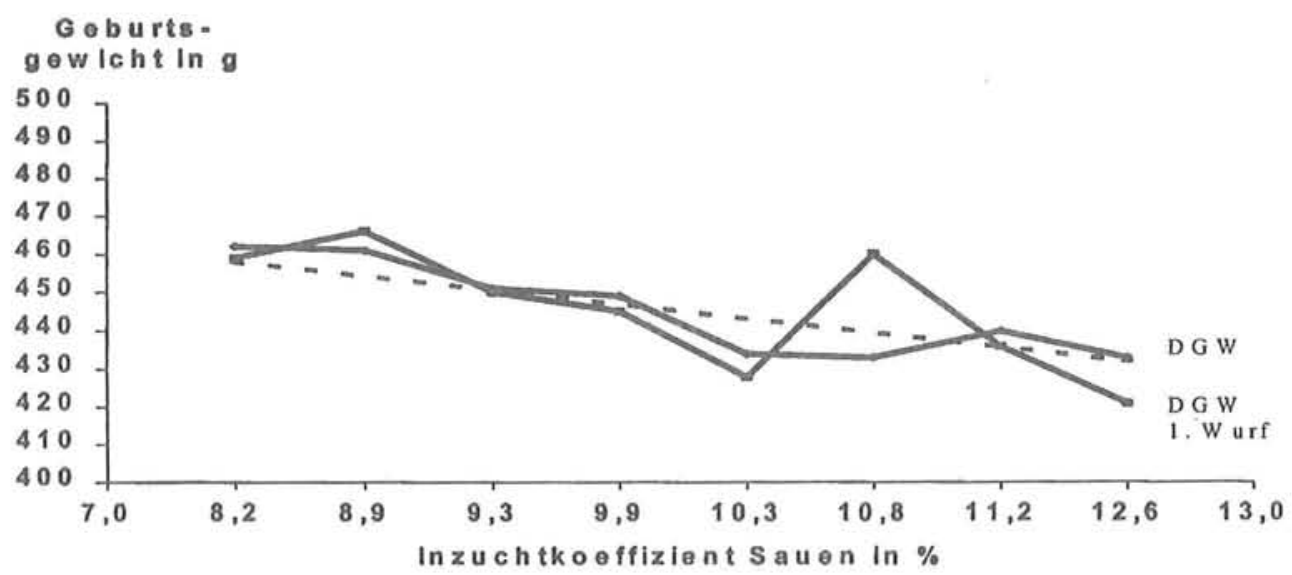

Abb. 4.: LSQ-Mittelwerte für das mittlere Geburtsgewicht für Inzuchtklasssen der Sauen (Werte auf der X-Achse stellen die mittlere Inzucht der jeweiligen Klasse dar) (Least square means for average birth weight for classes of inbreeding of sows) (values on the $\mathrm{x}$-axis represent the average inbreeding of sows within each class)

In Tabelle 7 sind die geschätzten Regressionskoeffizienten der Merkmale der Gewichtsentwicklung auf die Inzucht der Sauen und die Inzucht der Würfe bzw. Ferkel zusammen aufgeführt. Die Regressionskoeffizienten zeigen, daß die Zahl der Ferkel im Wurf den größten Einfluß auf das Geburtsgewicht und auch noch auf die Gewichtsentwicklung bis zum Alter von 6 Monaten hat.

Anhand der in Tabelle 7 dargestellten Regressionskoeffizienten wird auch deutlich, daß beim Geburtsgewicht, als Durchschnitt und auch als individuelles Geburtsgewicht, der Einfluß der Inzucht der Sau größer ist als der Einfluß der Inzucht der Ferkel. Bei der Gewichtsentwicklung in den ersten 6 Lebensmonaten zeigen beide Effekte eine sehr ähnliche Beziehung zum Gewicht. 
Tabelle 7

Geschätzte Regressionskoeffizienten der verschiedenen Gewichte auf den Inzuchtkoeffizienten (in Gramm pro \% Inzucht) und auf die Zahl der gesamt geborenen Ferkel pro Wurf (in Gramm pro Ferkel) mit den Standardfehlern der Regressionskoeffizienten in Klammern (Estimated regression coefficients of weights on the inbreeding coefficient (in gram per \% inbreeding) and on number of total piglets born within a litter (in gram per piglet) with standard errors of regression coefficients in brackets)

\begin{tabular}{l|cccc}
\hline & DGW & GGW & GEW06 & GEW12 \\
\hline Inzucht der Ferkel & $-2,3$ & $-1,3$ & $-25,6$ & $-88,5$ \\
Inzucht der Sau & $(1,32)$ & $(0,70)$ & $(6,7)$ & $(48,7)$ \\
& $-6,0$ & $-7,0$ & $-24,9$ & $-108,2$ \\
Gesamt geborene Ferkel & $(1,95)$ & $(0,97)$ & $(9,1)$ & $(85,4)$ \\
& $-10,9$ & $-11,0$ & $-48,2$ & $-36,7$ \\
& $(1,26)$ & $(0,64)$ & $(6,0)$ & $(49,1)$ \\
\hline DGW = durchschnittliches Geburtsgewicht & GGW & \# Geburtsgewicht GEW06 = Gewichtsabweichung bis 6 Mos
\end{tabular}

GEW12 = Gewichtsabweichung 6 bis 12 Monate

4. Diskussion

Bisherige Untersuchungen zur Inzuchtdepression bei Schweinen wurden entweder an Daten aus gezielten Inzuchtlinien mit steigenden Inzuchtkoeffizienten pro Jahr bzw. Generation (BERESKIN et al., 1968, 1970; MIKAMI et al., 1977) oder an Populationen mit sehr niedrigen mittleren Inzuchtkoeffizienten, über $50 \%$ nicht ingezüchtete Tiere und nur wenigen Tieren mit Inzuchtkoeffizeienten über $10 \%$ (TAKAHASHI et al., 1991) durchgeführt. Das vorliegende Datenmaterial spiegelt eher die Situation in kleinen geschlossenen Nucleuspopulationen kommerzieller Zuchtprogramme wider, in denen die Zuchtplanung auf die Minimierung der Inzucht ausgerichtet ist. Eine vergleichbare Untersuchung an kleinen geschlossenen Populationen wurde von TORO et al. (1988) und SILIO et al. (1994) an Iberischen Schweinelinien beschrieben, obwohl das Tiermaterial eine größere Streuung der Inzuchtkoeffizienten aufweist. Über mittlere Inzuchtkoeffizienten von geschlossenen Nucleuspopulationen kommerzieller Zuchtprogramme liegen keine Untersuchungen vor, es ist jedoch zu vermuten, daß die mittleren Inzuchtkoeffizienten niedriger sind als $10 \%$, wie in der hier untersuchten Population des Göttinger Minischweines. Da das hier untersuchte Datenmaterial nur aus einem Betrieb stammt und nur 6 Jahre an Daten ohne Inzuchtsteigerung (siehe Tab. 4) enthält, ist nicht mit den von LYNCH und WALSH (1998) beschriebenen statistischen Problemen der Schätzung der Inzuchtdepression zu rechnen.

Aus der Theorie über Inzucht und Heterosis ist Inzuchtdepression vorrangig bei niedrig erblichen, häufig mit der Fitness zusammenhängenden Merkmalen, wie z. B. der Wurfgröße zu erwarten. Die Ergebnisse von BERESKIN et al. $(1968,1970)$ und auch HILL und WEBB (1982) zeigen einen linearen Rückgang der Wurfgröße mit steigendem Inzuchtkoeffizienten, der aber kaum statistisch signifikant ist. In der vorliegenden Untersuchung wird bestätigt, daß weder der Inzuchtkoeffizient der Sau noch der Inzuchtkoeffizient des Wurfes eine erkennbare Beziehung zur Wurfgröße aufweist. Auch die Untersuchung von TORO et al. (1988) an Iberischen Schweinen mit mittleren Inzuchtkoeffizienten über $20 \%$ und einer großen Streubreite deuten die erwarteten negativen Regressionskoeffizienten der Wurfgröße auf die Inzucht der Sauen und der Ferkel an, die aber nur bei 
den größeren Linien Signifikanz zeigten. Wahrscheinlich reicht die durch höhere Inzucht verursachte Veränderung der intrauterinen Umwelt nicht aus, um die embryonale Sterblichkeit zu erhöhen und damit die Würfgröße signifikant zu senken.

Der hoch signifikante Einfluß der Inzucht der Sauen und der Ferkel auf das Geburtsgewicht und die spätere Gewichtsentwicklung wird in vielen Untersuchungen deutlich (BERESKIN et al., 1968, 1970; HILL und WEBB (1982) und SILIO et al., 1994), und auch in der vorliegenden Untersuchung bestätigt. Ausgedrückt als Prozent der phänotypischen Standardabweichung zeigt die Population des Göttinger Minischweines einen Rückgang im Geburtsgewicht von fast $70 \%$ pro $10 \%$ Steigerung des Inzuchtkoeffizienten der Sau und ca. $20 \%$ pro $10 \%$ Inzuchtsteigerung des Wurfes. Der größere Einfluß der Inzucht der Sau kann wahrscheinlich auf einen inzuchtbedingten Rückgang in der intrauterinen Versorgung der Embryonen zurückgefuhrt werden. In der Untersuchung von BERESKIN et al. (1968, 1970) macht die Inzuchtdepression auf das Geburtsgewicht nur 4 \% der phänotypischen Standardabweichung pro $10 \%$ Inzuchtsteigerung der Sau und $7 \%$ der phänotypischen Standardabweichung pro $10 \%$ Inzuchtsteigerung des Wurfes aus.

Auch in einer Untersuchung bei verschiedenen Schafrassen von ERCANBRACK und KNIGHT (1991) werden kaum signifikante Einflüsse der Inzucht der Lämmer und der Mutterschafe auf die Zahl der Lämmer wohl aber auf das Geburts- und Absetzgewicht beobachtet. In der Untersuchung ist auch zu beobachten, daß mit zunehmendem Alter der Lämmer der Einfluß ihrer Inzucht größer wird als der Einfluß der Inzucht ihrer Mütter. In der vorliegenden Untersuchung wird dieser Trend bestätigt. Während beim Geburtsgewicht eine 10-prozentige Steigerung der Inzucht der Mutter bezogen auf die phänotypische Standardabweichung einen mehr als 3 mal so hohen Effekt zeigte als eine 10-prozentige Steigerung der Inzucht des Wurfes, ist bis zum Alter von 6 Monaten pro $10 \%$ Steigerung der Inzucht des Wurfes oder der Sau ein identischer Rückgang für die Gewichtsentwicklung (27\% der phänotypischen Standardabweichung) zu beobachten. Für die Gewichtsentwicklung im Alter von 6 bis 12 Monaten zeigen die Inzucht der Sau und die Inzucht des Wurfes zwar auch ähnlich negative Effekte, die aber aufgrund des geringen Tiermaterials nicht statistisch abzusichern sind.

Die untersuchte Population des Göttinger Minischweins ist im Vergleich zu anderen Untersuchungen im Mittel weniger ingezüchtet und zeigt mit Inzuchtkoeffizienten von 5 bis $25 \%$ eine geringere Streubreite, sie spiegelt aber gut die Situation in geschlossenen Nucleuspopulationen in Zuchtunternehmen und auch vom Aussterben bedrohten kleinen Populationen wider. Langfristig ist aufgrund der hier gefundenen Ergebnisse davon auszugehen, daß in Schweinepopulationen mit steigender Inzucht kaum mit einem Rückgang in der Wurfgröße, aber mit einer signifikanten Reduktion der Geburtsgewichte und der späteren Gewichtsentwicklung zu rechnen ist. Mit einem negativen Einfluß reduzierter Geburtsgewichte auf die Aufzuchtverluste ist zu rechnen. Inwieweit die hier gefundenen Regressionskoeffizienten (auch prozentual in Einheiten Standardabweichungen ausgedrückt) auf andere Populationen zu übertragen sind, muß weiteren Untersuchungen vorbehalten bleiben. 


\section{Literatur}

BERESKIN, B., C. E. SHELBY, K. E. ROWE, W. E. URBAN, C . T. BLUNN, A. B. CHAPMAN, V. A. GARWOOD, L. N. HAZEL, J. F. LASLEY, W. T. MAGEE, J. W. MCCARTY AND J. A. WHATLEY : Inbreeding and swine productivity traits.J. Anim. Sci. 27 (1968), 339-350

BERESKIN, B.; SHELBY, C.E.; ROWE, K.E.; REMPEL, W.E.; DETTMERS, A.E.; NORTON, H.W.: Inbreeding and swine productivity in Minnesota experimental herds. J. Anim. Sci. 31 (1970), 278-288

BRANDT, H.; MÖLLERS, B.; GLODEK, P.: Prospects for a genetically very small minipig. Scandinavian Journal of Laboratory Anim. Sci. Suppl. 1, 1998, 93-96

ERCANBRACK, S. K.; KNIGHT, A.D.: Effects of inbreeding on reproduction and wool production of Rambouillet, Targhee and Columbia ewes. J. Anim. Sci. 69 (1991), 4734-4744

FALCONER, D. S.: Introduction to quantitative genetics. 3rd Ed. Longmann Sci. and Tech., Harlow, UK, 1989

GLODEK, P.; OLDIGS, B.: Das Göttinger Miniaturschwein. Schriftenreihe Versuchstierkunde 7 Paul Parey, 1981

HILL, W. G.; WEBB, A.J.:

Genetics of reproduction in the pig. In control of pig reproduction, (ed. D. J. A. Cole and G. R. Foxcroft), Butterworths, London, 1982, 541-564

LYNCH, M.; WALSH, B.:

Genetics and analysis of quantitative traits. Sinauer Associates, Inc. Publishers, Sunderland, Massachusetts 1997

MIKAMI, H.; FREEEEEDEEN, H.T.; SATHER, A.P.:

Mass selection in a pig population. 2. The effects of inbreeding within the selected populations. Can. J. Anim. Sci., 1977, 57, 627-634

SILIO, L.; RODRIGUEZ, M.; TORO, M.; RODRIGANEZ, J.:

Maternal and individual genetic effects on piglet weight. Proc. 5th World Congress on Genetics Applied to Livestock Produktion, Guelph, Canada 1994, Vol. 17, 355-358

TAKAHASHI, H.; CHRISTIAN, L.; ROTHSCHILD, M.; HARVILLE, D.; SUGIMOTO, T.:

Estimates of inbreeding depression of growth and backfat of Duroc pigs. Animal Science and Technology, 62 (1991), 323-329

TORO, M.; SILIO, L.; RODRIGANEZ, J.; DOBAO, M.:

Inbreeding and family index selection for prolificacy in pigs. Anim. Prod. 46 (1988), 79-85

Eingegangen: 09.08 .1999

Akzeptiert: 04.10.1999

Anschrin der Verfasser

Dr. HORST BRANDT, Dipl.-Ing, agr. BURCHHARD MÖLLERS

Institut fur Tierzucht und Haustiergenetik

der Universität Göttingen

Albrecht-Thaer-Weg 3

D-37075 Göttingen 